\title{
A SEMI-ANALYTICAL ISOGEOMETRIC ANALYSIS OF LEAKY WAVE PROPAGATION IN 3D WAVEGUIDES COUPLED WITH FLUIDS
}

\author{
Fakhraddin Seyfaddini $^{1,2}$, Hung Nguyen-Xuan ${ }^{3}$, Vu-Hieu Nguyen ${ }^{1,2}$ \\ ${ }^{1}$ Univ Paris Est Creteil, CNRS, MSME, F-94010 Creteil, France, \\ ${ }^{2}$ Univ Gustave Eiffel, MSME, F-77474 Marne-la-Vallée, France, \\ ${ }^{3}$ CIRTech Institute, Ho Chi Minh City University of Technology (HUTECH), \\ 475 Dien Bien Phu, P2, Binh Thanh, Ho Chi Minh City, Vietnam
}

Key words: Guided-waves, Dispersion curves, Isogeometric analysis, Semi-analytical finite element

\begin{abstract}
Guided waves are widely used as a non-destructive technique for the detection of defects or damage in structures due to the their excellent propagation characteristics. Developing an efficient calculation of guided waves is crucial in this context. In this work we present an approach named semi-analytical isogeometric analysis (SAIGA) for computation of dispersion properties of fluid-coupled waveguides in three-dimensional structures. The proposed approach is based on the use of Non-Uniform Rational B-splines (NURBS) as the basis functions for the geometry representation and for solution's approximation. It is shown that the using NURBS basis functions has significant advantage over using Lagrange basis functions for computing the dispersion of leaky waves in immersed waveguides due to their higher smoothness feature.
\end{abstract}

\section{INTRODUCTION}

Guided Ultrasonic Wave (GUW) technologies are widely used in the last decades as an efficient tool in the fields of Structural Health Monitoring and Nondestructive Evaluation, due to their capability of propagating for long distances while allowing the inspection of the entire cross-section of the waveguide. Due to the presence of boundaries and variation of material properties, the guided waves show a strong dispersive behavior, i.e. the phase velocity and attenuation vary with frequency-content of wave package. Therefore, the knowledge of the dispersion properties of GUWs is of fundamental importance in many practical applications. However, one of major issues in this problem is how to calculate efficiently the dispersion curves of all modes in the studied frequency range which will serve later to the inversion task. The Semi-Finite Element Method (SAFE) is one of most popular techniques for computing the dispersion of guided waves in structures thanks to its effectiveness in studying of functionally-graded or arbitrary cross-section waveguides $[1,2,3]$. However, the computational cost when using SAFE rapidly increases when we need to evaluate higher-modes and/or at higher frequencies. At high frequencies, using conventional high-order Lagrangian interpolation function does not allow to improve the situation because of numerical issues when solving eigenproblems.

The objective of this work is to study the effectiveness of using Non-Uniform Rational B-spline (NURBS) basis functions in the context of SAFE method for analyzing the wave propagation in $3 \mathrm{D}$ waveguides 
coupled with fluid. The results were compared with the ones obtained by analytical methods and by conventional SAFE method. Two cases of waveguides were investigated: (i) free loaded rail and (ii) fluidfilled cylinder. For both of cases, the dispersion curves evaluated by using enriched-NURBS method have a significant better precision than using conventional Lagrangian elements (for the same number of degrees of freedom), especially for the higher modes.

\section{Problem formulation}

In the following, the SAFE formulation is derived for a three-dimensional anisotropic elastic waveguide coupled with acoustic fluids. The section geometry and the mechanical properties of the structure are assumed to be uniform along the longitudinal direction, which is co-linear to the $\mathbf{x}_{3}$ of the Cartesian coordinate system $\left(\mathbf{x}_{1}, \mathbf{x}_{2}, \mathbf{x}_{3}\right)$. The idea is to solve the wave dispersion equations derived by applying the analytical method in the longitudinal direction and using finite element method in the cross-section surface of the considered waveguide.

\subsection{Governing equation}

\section{Geometry description The balance equations of linear momentum at a point $x \in \Omega^{s}$ and the linear} elastic constitutive law read

$$
\begin{aligned}
& \rho \ddot{u}_{s}-\nabla \cdot \sigma=0, \\
& \sigma=\mathrm{C} \varepsilon,
\end{aligned}
$$
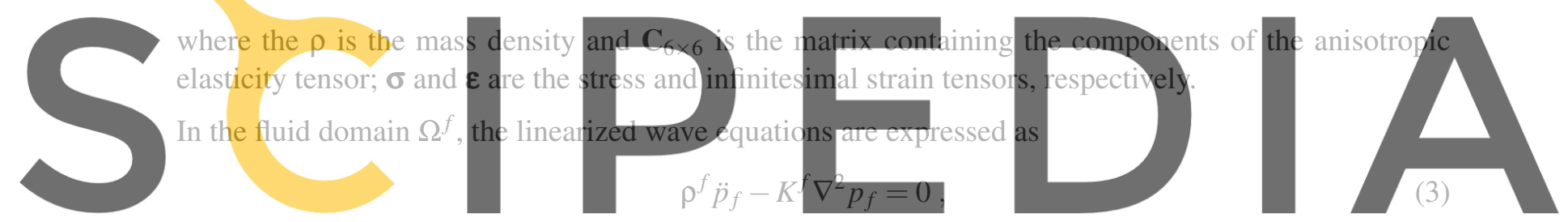

where $p_{f}$ are the acoustic pressure fields in $\Omega^{f}, K^{f}$ and $\rho^{f}$ are the bulk modulus and the mass density

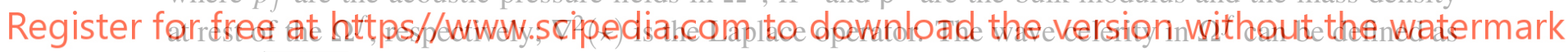
$c^{f}=\sqrt{K^{f} / \rho^{f}}$.

The boundary conditions of this system consist of the continuity condition of the normal displacement and traction at the solid-fluid interfaces $\Gamma^{s f}$ and the radiation condition at infinity, i.e.

$$
\left.\begin{array}{c}
\begin{array}{c}
\boldsymbol{u}_{s} \cdot \mathbf{n}^{s}=\boldsymbol{u}_{f} \cdot \mathbf{n}^{s} \\
\boldsymbol{t}
\end{array}=-p_{f} \mathbf{n}
\end{array}\right\} \quad \forall \boldsymbol{x} \in \Gamma^{s f},
$$

where $\boldsymbol{n}^{s}$ is the outward unit vector at the interfaces. It is worth to note that the outward unit vector of the solid domain is related to unit vector of the fluid domain by $\boldsymbol{n}^{s}=-\boldsymbol{n}^{f}$, and $\boldsymbol{t}=\boldsymbol{\sigma} \cdot \boldsymbol{n}^{s}$. We look for the solution of harmonic waves propagating along the axial direction $\left(\mathbf{e}_{3}\right)$ which may be expressed by the following form

$$
\begin{aligned}
& \boldsymbol{u}_{s}\left(x_{1}, x_{2}, x_{3}, t\right)=\boldsymbol{u}_{s}\left(x_{1}, x_{2}\right) e^{\mathrm{i}\left(k_{3} x_{3}-\omega t\right)} \\
& p_{f}\left(x_{1}, x_{2}, x_{3}, t\right)=p_{f}\left(x_{1}, x_{2}\right) e^{\mathrm{i}\left(k_{3} x_{3}-\omega t\right)}
\end{aligned}
$$


where $\mathrm{i}^{2}=-1 ; \omega \in \mathbb{R}$ is the angular frequency; $k$ is the wavenumber in the $\mathbf{e}_{3}$-direction; the vector $\boldsymbol{u}_{s}\left(x_{1}, x_{2}\right)=\left(u_{1}, u_{2}, u_{3}\right)^{T}$ and $p_{f}\left(x_{1}, x_{2}\right)=p_{f}$ which represents are the amplitudes of the displacement vector in the $\Omega^{s}$ and of the pressures in $\Omega^{f}$, respectively.

Noting that in the frequency-wavenumber $\left(\omega-k_{3}\right)$ domain, the time derivative and the spatial derivative with respect to $x_{1}$ can be replaced by: $(\star) \rightarrow-i \omega(\star)$ and $\partial_{1}(\star) \rightarrow \mathrm{i} k_{3}(\star)$, respectively.

\subsection{Weak formulation}

Upon integrating Eq. (1) against a test function $\delta \boldsymbol{u}$, applying the Gauss theorem and taking into account the interface conditions (4), the weak formulation of the boundary value problem in the solid layer $\Omega^{s}$ may be derived as:

$$
\int_{\Omega^{s}} \delta \boldsymbol{u}_{s} \cdot \rho \ddot{\boldsymbol{u}}_{s} d \Omega^{s}+\int_{\Omega} \nabla \delta \boldsymbol{u} \cdot \boldsymbol{\sigma} d \Omega^{s}+\int_{\Gamma^{s f}} \delta \boldsymbol{u}_{s} \cdot\left(p_{f} \boldsymbol{n}^{s}\right) d \Gamma^{s f}=0, \quad \forall \delta \boldsymbol{u}_{s} \in \mathcal{C}^{a d} .
$$

For the inner fluid domain $\Omega^{f}$, by introducing the test function $\delta p_{f}$, the weak formulation of the wave equation may be derived from (Eq. (3)):

$$
\int_{\Omega^{f}} \delta p_{f}^{*} \rho^{f} \ddot{p}_{f} d \Omega^{f}+\int_{\Omega^{f}} \nabla \delta p_{f} K^{f} \nabla p_{f} d \Omega^{f}+\int_{\Gamma^{s f}} \delta p_{f}^{*} \rho^{f} K^{f} \ddot{u}_{s} \cdot n^{s} d \Gamma^{s f}=0, \quad \forall \delta p_{f} \in C^{a d} .
$$

\section{Solution approximation using NURBS basis functions}

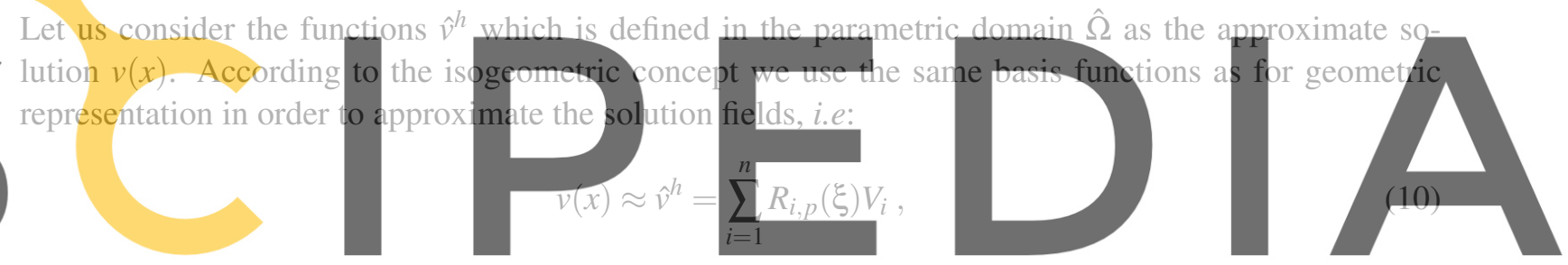

where the coefficients $V_{i} \in \mathbb{C}$ are the corresponding control variables (values at the control points). The

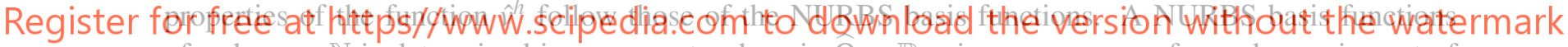

of order $p \in \mathbb{N}$ is determined in a parameter domain $\Omega \subset \mathbb{R}$ using a sequence of non-decreasing set of coordinates called knot vector defined as $\Xi=\left\{\xi_{1}, \xi_{2}, \xi_{3}, \ldots, \xi_{i+p+1}\right\}$, where $\xi_{i} \in \mathbb{R}$ is the $i^{\text {th }}$ knot, $i$ is the

knot index, $i=1,2, \ldots, n+p+1$, and $n \in \mathbb{N}$ is the number of basis functions used to construct the B-spline curve. Given a knot vector, the B-spline basis functions are defined recursively starting for $p=0$ as

$$
N_{i, 0}(\xi)= \begin{cases}1 & \text { if } \xi_{i}<\xi<\xi_{i+1}, \\ 0 & \text { otherwise }\end{cases}
$$

and for $p=1,2,3, \ldots$, they are defined by the well-known Cox-de Boor recursion formula as

$$
N_{i, p}(\xi)=\frac{\xi-\xi_{i}}{\xi_{i+p}-\xi_{i}} N_{i, p-1}(\xi)+\frac{\xi_{i+p+1}-\xi}{\xi_{i+p+1}-\xi_{i+1}} N_{i+1, p-1}(\xi) .
$$

Thus, non-uniform rational B-spline (NURBS) basis functions are built from the B-spline functions by multiplying weighting functions $w_{i}$ for each basis

$$
R_{i, p}(\xi)=\frac{N_{i, p}(\xi) w_{i}}{\sum_{j=1}^{n} N_{j, p}(\xi) w_{j}},
$$


where $w_{i}$ denotes the weight value associated with control point vector $P_{i}$. The NURBS basis functions have some advantage such as higher continuity across the element boundaries, partition of unity, variation diminishing, linear independence and compact support. Using the inversion of geometrical mapping $x(\xi, \eta)$ the function $\hat{v}^{h}$ over the physical domain $\Omega$ can be define such that $v^{h}=\hat{v}^{h} \circ x^{-1}$.

In this study, the Galerkin finite element method is employed, in which the same approximations are applied for both functions $\boldsymbol{u}_{s}^{h}$ and $\delta \boldsymbol{u}_{s}^{h}$ (as well as for $p_{f}^{h}$ and $\delta p_{f}^{h}$ ) on each patch:

$$
\begin{array}{cc}
\boldsymbol{u}_{s}^{h}=\mathbf{R}^{u} \mathbf{U}_{s}, & \delta \boldsymbol{u}^{h}=\mathbf{R}^{u} \delta \mathbf{U}_{s}, \\
p_{f}^{h}=\mathbf{R}^{p} \mathbf{P}_{f}, & \delta p_{f}^{h}=\mathbf{R}^{p} \delta \mathbf{P}_{f},
\end{array}
$$

where $\mathbf{R}^{u}, \mathbf{R}^{p}$ are the interpolation matrix containing the NURBS basis functions (Eq. (12)); $\mathbf{U}$ and $\delta \mathbf{U}$ are the vectors of control displacements; $\left(\mathbf{P}_{f}\right)$ and $\left(\delta \mathbf{P}_{f}\right)$ are the displacement vectors of control pressures. In this paper we consider the same NURBS basis functions for approximation of the solution fields in Eqs. (13a)-(13b). By substituting the approximations Eqs. (13a)-(13b) into the weak formulations Eqs. (8)-(9), then assembling the elementary matrices, one obtains

$$
\left(-\omega^{2} \mathbf{M}+\mathbf{K}_{0}+\mathrm{i} k_{3} \mathbf{K}_{1}+k_{3}^{2} \mathbf{K}_{2}\right) \mathbf{V}=\mathbf{0},
$$

where $\mathbf{V}=\left(\mathbf{U}_{s}, \mathbf{P}_{f}\right)^{T}$ containing the global eigenvectors of pressure $\left(\mathbf{P}_{f}\right)$ and of displacement $\left(\mathbf{U}_{s}\right)$; the global matrices $\mathbf{M}, \mathbb{K}_{0}, \mathbb{K}_{1}, \mathbb{K}_{2}$ are defined by:
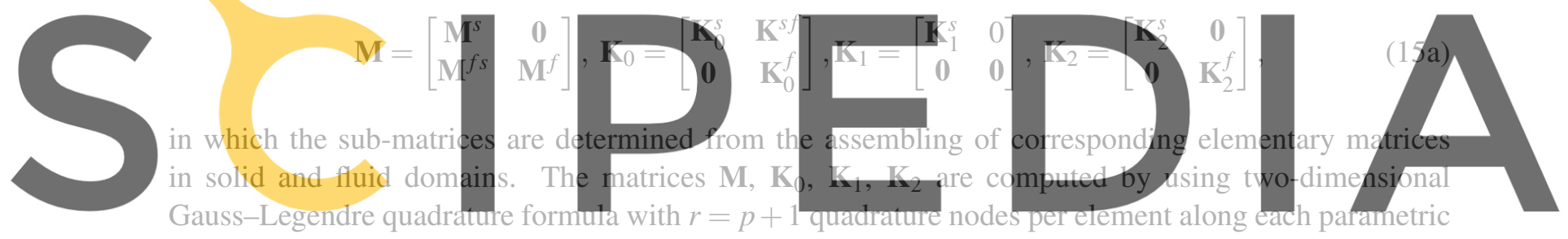

direction which has been shown to be efficient [4]

Register for free at https//www.scipedia.com to download the version without the watermark The system of characteristic equations (14) is an elgenvalue problem which is used to determine the relationship between the pulsation $\omega$ and the wavenumber $k_{3}$. For each value of the angular frequency $\omega$, solving Eq. (14) allows us to determine the eigenvalues $k_{3}$ and their associated eigenvectors (also called by wave structures), $\mathbf{V}\left(\omega, k_{3}\right)$ of guided modes. The frequency-dependent phase velocity $\left(C_{\mathrm{ph}}\right)$ and the attenuation (att) of a guided mode are given by:

$$
C_{\mathrm{ph}}=\frac{\omega}{\operatorname{Re}\left(k_{3}\right)}\left[\mathrm{m} \cdot \mathrm{s}^{-1}\right], \quad \text { att }=\operatorname{Im}\left(k_{3}\right)\left[\mathrm{Np} \cdot \mathrm{m}^{-1}\right],
$$

where $\operatorname{Re}()$ and $\operatorname{Im}()$ denote the real and imaginary parts of a complex function.

\section{Numerical examples}

\subsection{Waveguide with arbitrary cross-sections: railroad track}

The purpose of this section is to demonstrate the robustness of the SAIGA to analyze the wave propagation in waveguides of arbitrary cross-sections such as rail. The case considered is a typical railroad track 115-lb A.R.E.M.A shown in Fig. 1. The analysis of dispersion behavior and mode shapes are usually 
employed for studies of non-destructive defect detection and of the deduced-vibration noise in the rails. For this example, the rail's material is assumed as an isotropic material with the density $\rho=7932 \mathrm{~kg} / \mathrm{m}^{3}$, the longitudinal bulk wave velocity $c_{L}=5960 \mathrm{~m} / \mathrm{s}$, the shear bulk wave velocity $c_{T}=3260 \mathrm{~m} / \mathrm{s}$.

Due to the geometrical complexity of the rail cross section, the analytical solution to the wave propagation in the rail is not feasible. Here, we present and compare the numerical solutions obtained by the conventional SAFE and SAIGA methods, of the meshes are shown in Fig. 1. Due the the irregular form of the rail's section, the conventional SAFE method need to use a very fine mesh to correctly represent the corners.

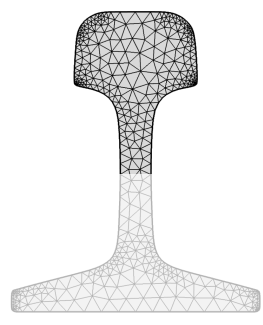

(a)

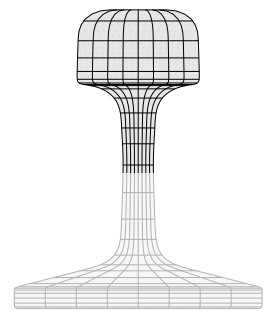

(b)

Figure 1: Rail section meshing: (a) Mesh with 883 tringular elements (total of 5868 degree of freedom) using conventional SAFE, (b) NURBS-based mesh with 210 elements (total of 1220 degree of freedom) using 5 order

NURBS basis functions.

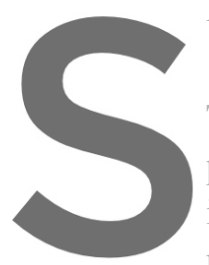

The results are shown plotted in Fig. 2 reveal the in rail. The IGA allows results compare with conventionat SAFE method.

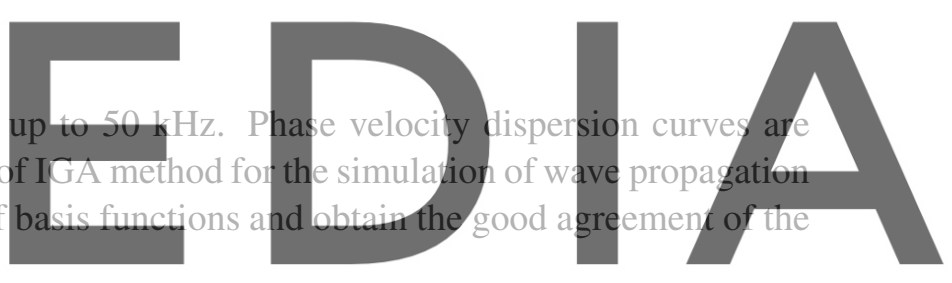

Register for free at https//www!ßeqpedia.com to download the version without the watermark

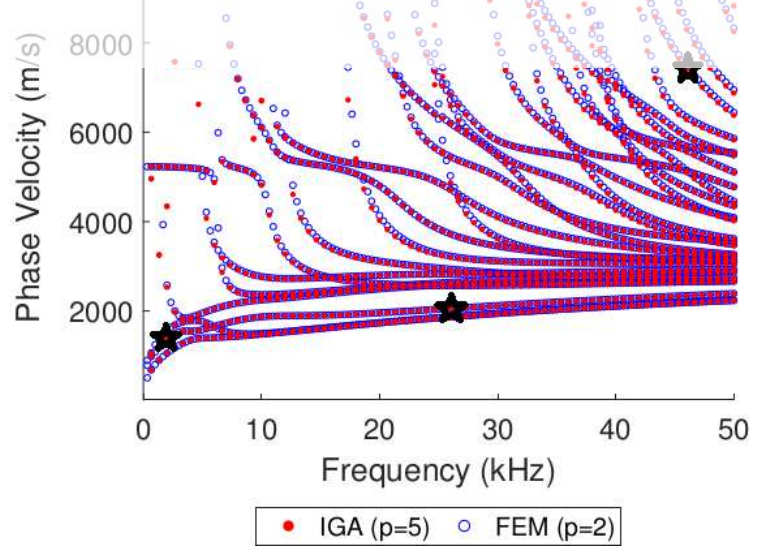

Figure 2: Dispersion curves of 115-lb A.R.E.M.A. rail using IGA-based SAFE (red marker), and conventional SAFE implemented in Comsol Multiphysics (blue marker) for 5 order NURBS basis functions. 
We examine the mode shape of the rail by choosing different value of frequency for the low and high frequency domain. The Fig. 3 shows the mode shapes at several frequencies which correspond to points marked by black stars shown in Fig. 2). These modes excite preferably a certain portion of the rail. This information can be used in practical NDE tests to target defects at various locations in the rail section. Similarly, knowledge of the mode shapes is necessary to design the appropriate wave excitation/detection approach. It can be observed SAIGA results of the considered mode shape, which are computed by using $\mathrm{p}=5$ and $N_{d o f}=1220$ have good agreements with the SAFE computed using $N_{d o f}=5868$. These results show that NURBS allows to obtain the modes at high frequencies using less degree of freedom compared to the conventional method.

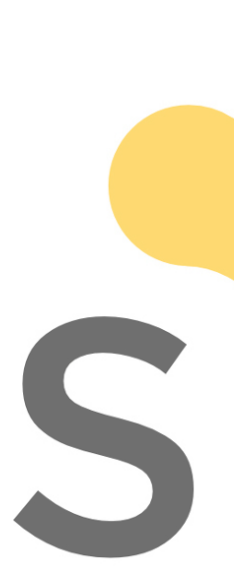

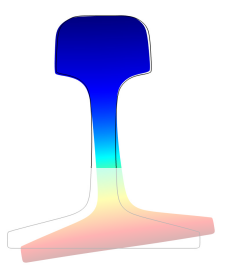

(a) SAFE (2 kHz)

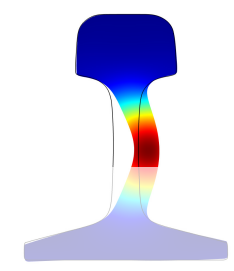

(b) SAFE $(26 \mathrm{kHz})$

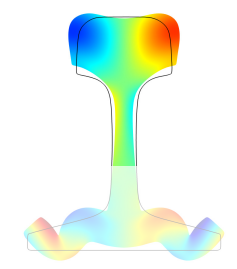

(c) SAFE $(46 \mathrm{kHz})$

\section{Register for free at https//www.scipedia.com to download the version without the watermark} 4.2 Dispersion of guided-waves in fluid-filled cylinder

Guided-wave propagation in a steel cylinder filled by water, as shown in Fig. 4(a), is considered in this example. The steel cylinder has the same geometry and material properties as the one studied in the previous section. The acoustic properties of water are given by the density $\rho^{f}=998 \mathrm{~kg} \cdot \mathrm{m}^{-3}$ and the sound speed $c^{f}=1478 \mathrm{~m} \cdot \mathrm{s}^{-1}$. The outer surface of the cylinder is assumed to to be free.

In Fig. 4(b), we compare the numerical solutions of $C_{\mathrm{ph}}$ obtained by SAIGA method to the analytical ones over the frequency range from 0 to $1 \mathrm{MHz}$. The discretization is performed by using cubic NURBS basis functions. The total number of degrees of freedom for SAIGA model equals to $N_{\text {dof }}=1041$. A filtering procedure as the presented in the previous section was applied to extract the modes of interest according to their symmetries. It is shown that the dispersion curves computed by SAIGA method agree well with the analytical dispersion ones. 


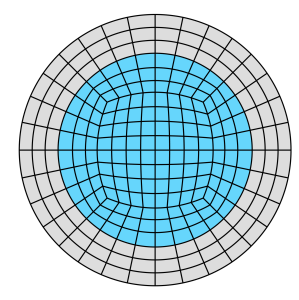

(a) SAIGA $\left(N_{\text {dof }}=1041\right)$

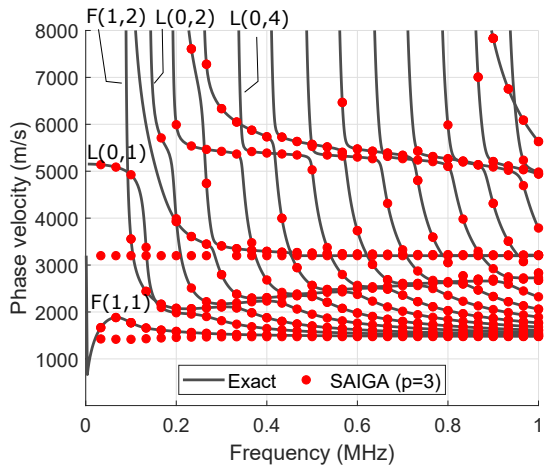

(b) SAIGA $p=3, N_{\mathrm{dof}}=1041$

Figure 4: Dispersion curves of a fluid-filled steel cylinder: comparison between the analytical solution (grey line) and SAIGA solution (red marker)

\section{Conclusion}

Due to the strong frequency dependency of dispersion properties, determination of guided waves at high frequency range often requires high computational cost. The dispersion curves based on the SAIGA showed the better accuracy results compared to the conventional SAFE method. The number of degrees of freedom using SAIGA can be significantly reduced in comparing with the ones required by using

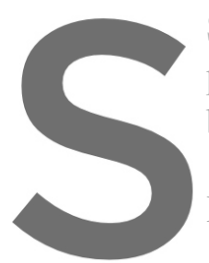
SAFE method to achie

produces more accuracy be extended to other proble

REFERENCES

[1] T.N.H.T. Tran, L.H. Le; M. D. Sacchi, V.-H. Nguyen, Sensitivity analysis of ultrasonic guided

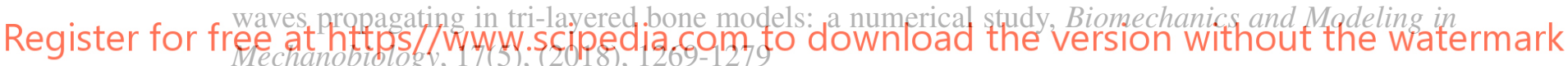

[2] M. Mazzotti and A. Marzani and I. Bartoli, Dispersion analysis of leaky guided waves in fluidloaded waveguides of generic shape, Ultrasonics 54 (1) (2014) 408-418.

[3] D. Pereira, G. Haiat, J. Fernandes, P. Belanger, Simulation of acoustic guided wave propagation in cortical bone using a semi-analytical finite element method, The Journal of the Acoustical Society of America 141 (4) (2017) 2538-2547.

[4] F. Seyfaddini, H. Nguyen-Xuan, V.-H. Nguyen, A semi-analytical isogeometric analysis for wave dispersion in functionally graded plates immersed in fluids, Acta Mechanica 232 (2021) 15-32.

[5] V.-H. Nguyen, S. Naili, Simulation of ultrasonic wave propagation in anisotropic poroelastic bone plate using hybrid spectral/finite element method, International Journal for Numerical Methods in Biomedical Engineering 28 (8) (2012) 861-876. 